\title{
Supersymmetry breaking in heterotic strings and the decompactification problem
}

\section{loannis Florakis*}

Laboratoire de Physique Théorique et Hautes Énergies - LPTHE

Sorbonne Université, CNRS, 4 Place Jussieu, 75005 Paris, France

E-mail: florakis@lpthe.jussieu.fr

\begin{abstract}
We review recent progress in addressing the decompactification problem of gauge couplings in the context of heterotic string theory with spontaneous supersymmetry breaking. This problem is particularly relevant in large volume scenarios where quantum corrections to gauge couplings typically explode and the theory is driven to the non-perturbative regime. We discuss a recently proposed mechanism in which the problem may be avoided by balancing the contribution of Kaluza-Klein and winding states against the universal contribution due to gravitational and dilaton diagrams.
\end{abstract}

Corfu Summer Institute 2017 'School and Workshops on Elementary Particle Physics and Gravity' 2-28 September 2017

Corfu, Greece

${ }^{*}$ Speaker. 


\section{Spontaneous supersymmetry breaking in heterotic string theory}

Supersymmetry has been traditionally considered as one of the central ingredients of string theories. From the point of view of the $2 \mathrm{~d}$ string worldsheet, local $\mathscr{N}_{2}=1$ superconformal symmetry plays a central role in removing unphysical degrees of freedom and securing the correct implementation of spacetime fermions in the spectrum of the theory. Although strings with spacetime supersymmetry have been the subject of extensive study over the last few decades, the study of supersymmetry breaking in string theory is not a new subject [1, 2, 3, 4, 5, 6, 7, 8, 9, 10, 11, 12]. Already from the early days of string theory, various aspects of supersymmetry breaking were considered with prime examples the study of strings at finite temperature $[13,14]$ and the $\mathrm{O}(16) \times \mathrm{O}(16)$ theory [15].

Nevertheless, non-supersymmetric string theories have not been exhaustively studied, partly due to technical difficulties associated with the problem of taming their radiative corrections. More recently, in the context of string phenomenology, there has been an increased interest in nonsupersymmetric string constructions [16, 17, 18, 35, 19, 20, 21, 22, 23, 24, 25, 26, 27, 28, 29, 30] and, in particular, there has been considerable progress in understanding radiative corrections to gauge and gravitational couplings in such setups. Part of this progress was possible thanks to the development of new mathematical techniques for studying string loop amplitudes [31, 32, 33, 34, $35,36]$.

As soon as supersymmetry is broken in string theory, various terms in the effective action that were previously protected now begin receiving quantum corrections already at one loop. This includes, in particular, the scalar potential of the theory. Since these terms are no longer BPS saturated, one typically expects all perturbative states of the string to run in the loops, including oscillator as well as Kaluza-Klein (KK) and winding states.

In this way, the entire tower of heavy string states contributes to loop corrections and induces a dependence on the compactification moduli, which is expected to play a significant role in any quantitative comparison with low energy data. For instance, in the case of gauge couplings, the one loop correction

$$
\frac{16 \pi^{2}}{g^{2}(\mu)}=\frac{16 \pi^{2}}{g^{2}\left(M_{s}\right)}+b \log \frac{M_{s}^{2}}{\mu^{2}}+\Delta
$$

the effect of the heavy string modes is encoded in the threshold correction $\Delta$, which is a function of the compactification moduli of the theory.

Whenever supersymmetry is broken in string theory one is asked to address at least two fundamental questions, related to the possible destabilisation of the vacuum at tree and loop level. The first concerns the possible development of tachyonic excitations in the theory's spectrum. This is present both in cases where the breaking is explicit as well as when it is spontaneous. This is closely related to the exponential growth in the number of massive states leading to Hagedorn divergences. The second question concerns the destabilisation of the classical vacuum by loop effects, in the form of back-reaction that already is present at one-loop (c.f. [37, 38]).

Some special constructions do exist in the literature, for instance, removing tachyonic excitations by turning on fluxes in certain setups. Another interesting possibility would be to project them out by means of some asymmetric orbifold action [16, 39], possibly related to non-geometric 
fluxes $[40,11]$. We shall not venture into this direction, but instead discuss dynamical mechanisms that protect the theory against such possibilities.

A special way to break supersymmetry in string theory, which has the benefit of retaining perturbative calculability, is the Scherk-Schwarz mechanism [41, 42, 43, 44, 1, 2, 3]. It corresponds to a flat gauging of supergravity in the field theory limit, whereas from the worldsheet perspective, it can be reformulated as a freely acting orbifold. A simple way to illustrate the Scherk-Schwarz mechanism in field theory, is to see it as a deformation of the theory such that certain fields that were previously periodic as they encircle a compact dimension, now become periodic only modulo a symmetry operation generated by a charge $Q$. For instance, in the case of a scalar field, $\Phi\left(X_{5}+\right.$ $2 \pi R)=e^{2 \pi i Q} \Phi\left(X_{5}\right)$. This has the effect of shifting the Kaluza-Klein spectrum of charged states and, in particular, introduces a mass gap

$$
M_{\mathrm{KK}}=\frac{|Q|}{R} .
$$

If the operator $Q$ is identified with the spacetime fermion number $F$, bosons and fermions within the same multiplet are essentially assigned different boundary conditions and, hence, different masses. The result is a spontaneous breaking of supersymmetry with a breaking scale inversely proportional to the compactification radius.

One of the important properties of Scherk-Schwarz breaking, related ultimately to its worldsheet tractability in the string case, is the no-scale structure of the scalar potential. Indeed, as soon as one minimises the tree-level potential with respect to the charged states, the potential vanishes and the scale of supersymmetry breaking remains undetermined at tree level. The size and shape of the compactified dimensions participating in the Scherk-Schwarz mechanism therefore remain moduli at tree level.

Nevertheless, one may expect that loop corrections to the scalar potential introduce non-trivial dependence on the no-scale moduli which might stabilise, or even destabilise them. This opens the possibility for a dynamical determination of the supersymmetry breaking scale. The main question, in such cases, is naturally related to the morphology of the one-loop effective potential in such theories, and which ingredients mostly affect its shape.

Since fixed points under stringy symmetries (T-dualities) naturally translate into (local) extrema of the potential and the natural scale of the problem is the string scale, one expects a typical form of the effective potential to be either in the shape of a well, or a lump, with an extremum close to the string scale. In addition, for large radii, the gravitino mass scale becomes sufficiently small and supersymmetry is asymptotically recovered in the infinite radius limit. We, therefore, expect the potential to vanish in the same limit.

In most cases of Scherk-Schwarz breaking, and provided one is working in some sub-region of moduli space where no tachyons are encountered, the one-loop corrected potential takes the form of a well, with its minimum at negative values of the potential, trapping the no-scale modulus to $R \sim 1$ in string units. In other words, supersymmetry is broken at the string scale, which clearly is not a scenario relevant for discussing the hierarchy problem. Moreover, this situation is extremely unstable, since the negative potential at the minimum generates a huge negative cosmological constant. Furthermore, since the modulus is now trapped around the string scale, small perturbations may lead to the excitation of tachyonic modes and a full stability analysis is required. In such cases, there typically always exists a deformation direction along which winding tachyons will emerge. 
A much more interesting situation is if the loop-corrected potential has the shape of a bump, with a maximum again around the fixed points but at positive values for the potential. Provided no minimum is established at negative values of the potential, this shape typically triggers spontaneous decompactification and the radius of the compact dimension participating in the Scherk-Schwarz breaking dynamically rolls to very large values. Essentially, the theory tends asymptotically towards the supersymmetric regime, with the gravitino mass $m_{3 / 2} \sim 1 / R$ becoming smaller and smaller. Provided another mechanism is at play, possibly due to non-perturbative effects, which stabilises the radius field to some phenomenologically desirable value, the possibility is opened for supersymmetry breaking at low scales, possibly comparable to the $\mathrm{TeV}$ range. An additional benefit of this scenario is that, with the radius being driven away from the string scale, the theory is also dynamically protected against tachyonic excitations. This is a generic feature of large volume scenarios [45].

There are, however, additional constraints to consider. The generic asymptotic behaviour of the potential at large radii is of the form $V_{\text {loop }} \sim\left(n_{F}-n_{B}\right) / R^{4}$, where $n_{F, B}$ is the number of massless fermionic/bosonic states in the theory. Setting the breaking scale to be around the $\mathrm{TeV}$ range, the resulting potential is still too large compared to the observed value for the cosmological constant; in fact, overshooting it by nearly 40 orders of magnitude.

A possible solution to this problem was already suggested by Antoniadis in [45], by imposing a bose-fermi degeneracy $n_{F}=n_{B}$ to the massless states in the string spectrum. In this case, the leading polynomial $1 / R^{4}$ fall of the potential is eliminated, and the potential then exhibits an exponential suppression consistent with the requirements for a small vacuum energy at large volume. Such models, precisely because they almost carry the no-scale property also to the loop level, were termed 'super no-scale' models [21, 28, 29]. In addition to allowing an exponentially small value for the cosmological constant, they also naturally induce an exponentially small back-reaction.

An important question that arises next is whether it is actually possible to construct actual string models with chiral matter, which realise the above large-volume scenario. It turns out that it is, in fact, possible and the first such examples within the framework of heterotic string theory were given in [27]. In the same work, it was found that the super no-scale condition by itself is not sufficient to guarantee the desired shape of the potential. Non-level matched states around selfdual points actually do significantly affect the shape of the potential, including its sign. The precise conditions can be found, e.g. at the fermionic point, in [27].

Another interesting result concerns the dependence of the potential on other 'orthogonal' moduli, not directly participating in the Scherk-Schwarz breaking. An investigation in [27] indicates that these tend to be stabilised at values of the order of the string scale, at least for sufficiently large radii. Although the explicit models constructed in [27] leave an unbroken SO(10) GUT group, they still lay the ground for further investigation in more realistic setups.

Having outlined the possibility for naturally triggered large volume scenarios in heterotic strings, we can now return to the question of radiative corrections to gauge and gravitational couplings. Threshold corrections to such couplings in the string effective action were extensively studied $[46,47,48,49,50,51,52,53,54,55,56,57]$ throughout the 90 's in the case of at least one unbroken supersymmetry, with several seminal results. Nevertheless, very little was known about how such couplings renormalise in non-supersymmetric string theory.

The calculation of gauge threshold corrections involves the scattering amplitude of two gauge 
bosons at one loop,

$$
\frac{16 \pi^{2}}{g^{2}}=\int_{\mathscr{F}} \frac{d^{2} \tau}{\tau_{2}^{2}} \int_{\text {torus }} d^{2} z\left\langle\mathscr{V}^{a}(z, \bar{z}) \mathscr{V}(0)\right\rangle_{\mathrm{CFT}}
$$

After evaluating the correlator of the gauge bosons for the genus-1 worldsheet CFT, one is instructed to integrate over their relative position $z$ on the worldsheet torus, and eventually perform an integral over all gauge-inequivalent shapes of the torus, parametrised by the complex structure parameter $\tau$, over the fundamental domain $\mathscr{F}$.

A seminal result due to Dixon, Kaplunovsky and Louis [47] gives rise to the notion of supersymmetric universality. Focusing on the dependence on the compactification moduli, for the case of unbroken supersymmetry, the difference of gauge thresholds for two different gauge group factors takes the form

$$
\Delta_{1}-\Delta_{2}=b_{12} \int_{\mathscr{F}} \frac{d^{2} \tau_{2}}{\tau_{2}^{2}} \sum_{\text {states }} e^{-\pi \tau_{2} M^{2}}=-b_{12} \log T_{2} U_{2}|\eta(T) \eta(U)|^{4} .
$$

The sum is over BPS states, and the dependence on the compactification moduli $T, U$ enters through the BPS mass $M^{2}$, which encodes the contributions of Kaluza-Klein momentum and winding states. The prefactor $b_{12}$ is simply the difference between the beta function coefficients for the two gauge groups in question, associated to the massless spectrum of the theory. It is remarkable that this modular integral can be exactly evaluated in closed form, in terms of the logarithm of Dedekind $\eta$ functions. It is impressive that for a large class of constructions, the above result is largely universal. The model dependence enters only through the multiplicative prefactor $b_{12}$.

It is highly non-trivial that a similar version of universality exists also in heterotic theories with spontaneously broken supersymmetry à la Scherk-Schwarz introduced as a momentum shift along the first cycle of a $T^{2}$ of internal space. Under certain precise conditions given in [20], for a large class of such theories, the difference of gauge thresholds can be again cast into a universal closed form [17]

$$
\begin{array}{r}
\Delta_{1}-\Delta_{2}=\alpha \log T_{2} U_{2}|\eta(T) \eta(U)|^{2} \\
+\beta \log T_{2} U_{2}\left|\vartheta_{4}(T) \vartheta_{2}(U)\right|^{2} \\
+\gamma \log \left|\hat{j}_{2}(T / 2)-\hat{j}_{2}(U / 2)\right|^{4}\left|j_{2}(U)-24\right|^{4},
\end{array}
$$

where $\vartheta_{2}$ and $\vartheta_{4}$ are the well known Jacobi theta constants, $j_{2}$ is the Hauptmodul of the Hecke congruence subgroup $\Gamma_{0}(2) \in \operatorname{SL}(2 ; \mathbb{Z})$ and $\hat{j}_{2}$ is obtained from $j_{2}$ by the Fricke involution $\tau \rightarrow$ $-1 /(2 \tau)$. This highly unexpected result indicates again a form of non-supersymmetric universality in differences of gauge thresholds. Indeed, the only model dependence enters into the coefficients $\alpha, \beta, \gamma$ which have a precise interpretation as differences of beta-function coefficients (in general, for subsectors of the theory) and may be again computed from the knowledge of the massless spectrum and its quantum numbers alone.

A generalised version of the above universal expression can also be obtained [25] for the gauge threshold corrections themselves (as opposed to only their differences) and also for gravitational thresholds, by exploiting the symmetries under congruence subgroups of the modular group, although it cannot be cast in a simple closed form. 
One expects that in the large volume limit, gauge thresholds are dominated by the $6 \mathrm{~d}$ limit of the theory. In $6 \mathrm{~d}$, however, the gauge coupling scales with squared length and so, we expect thresholds to behave as $\Delta_{a} \sim \tilde{\beta}_{a} T_{2}$, where $T_{2}$ is the volume of the compactification 2-torus along which Scherk-Schwarz is acting as a translation. Here, $\tilde{\beta}_{a}$ is the beta function coefficient for the massless degrees of freedom surviving in the $6 \mathrm{~d}$ limit of the theory charged under the gauge group factor $G_{a}$. By inspecting the above universal expressions for threshold differences, it is straightforward to see that this is indeed the case.

At large volume, the thresholds receive a huge contribution (in magnitude) from the term involving the Dedekind $\eta$-functions; a behaviour present both in the supersymmetric as well as the non-supersymmetric case. If the beta function coefficient for the gauge group in question is positive, then the one loop-corrected gauge coupling becomes extremely small and effectively decouples. If, on the other hand, $\tilde{\beta}_{a}<0$, the large volume contribution quickly drives the theory to the strong coupling regime and ruins the theory's predictability at lower energies. This issue is known as the decompactification problem and appears to be generic in heterotic theories at large volume.

Some ways to solve the decompactification problem have appeared in the literature $[51,18]$. The idea is to modify the construction such that a theory with 16 supercharges is asymptotically recovered in the large volume (6d) limit of the theory $T_{2} \rightarrow \infty$. In other words, as $T_{2} \rightarrow \infty$ the theory is effectively dominated by the $\mathscr{N}=4$ sector, instead of an $\mathscr{N}=2$ one. This suppresses the contribution of the heavy modes and removes the problematic $\log |\eta(T)|$-terms, and replaces them with Jacobi theta functions, i.e. $\log \left|\vartheta_{4}(T)\right|$ which are suppressed at large volume. This solves the decompactification problem. Unfortunately, it does not appear to be possible to realise this scenario for a theory with chirality.

An alternative way of looking at the decompactification problem [30] is to view it not as a problem but, rather, as a vacuum selection criterion. It is possible, in fact, to impose conditions on the theory such that the decompactification problem is absent. This approach is compatible with chirality and it is this viewpoint that we wish to adopt here.

Let us first note that it is possible to decompose the large volume behaviour of gauge thresholds into two parts

$$
\Delta_{a}=-\frac{k_{a}}{48} Y+\hat{\beta}_{a} \Delta+\ldots,
$$

in which the first two terms constitute the $\mathscr{N}=2$ subsector of the theory, that governs the $6 \mathrm{~d}$ limit of the theory. The remaining $\mathscr{N}=1$ and $\mathscr{N}=4$ subsectors are absorbed in the ellipses, and $k_{a}$ is the Kac-Moody level for a given gauge group factor $G_{a}$. The first term is a universal part that can be traced to diagrams involving the universal coupling of graviton and dilaton to the gauge fields, while the second term is the actual running part multiplied by the $\mathscr{N}=2$ sector beta function coefficient of the $6 \mathrm{~d}$ theory. The ellipses denote terms that grow at most logarithmically at large volume. Typically, one does not deal with the universal part $Y$, since it can be absorbed into a redefinition of the tree level coupling. One is then usually left only with the running part

$$
\hat{\beta}_{a} \Delta=-\hat{\beta}_{a} \log T_{2} U_{2}|\eta(T) \eta(U)|^{4}=\hat{\beta}_{a}\left(\frac{\pi}{3} T_{2}-\log T_{2}\right)+\ldots
$$

Indeed, as discussed before, the effect of the running part $\Delta$ is to subtract the $4 \mathrm{~d}$ logarithmic growth and replace it with the $6 \mathrm{~d}$ polynomial growth. This leads to the decompactification problem. 
The key observation now is that not only does the running part $\Delta$ grow linearly with the compactification volume $T_{2}$, but so does the universal part $Y$, which is defined by the modular integral

$$
Y=\int_{\mathscr{F}} \frac{d^{2} \tau}{\tau_{2}} \Gamma_{2,2}(T, U)\left(\frac{\hat{E}_{2} E_{4} E_{6}-E_{4}^{3}}{\eta^{24}}+1008\right) .
$$

Here, $\hat{E}_{2}$ is the quasi-holomorphic Eisenstein series of weight 2, while $E_{4}, E_{6}$ are the holomorphic Eisenstein series of weight 4 and 6, respectively. By carefully unfolding and evaluating this integral in the large volume limit, one finds indeed that the universal part grows linearly with the volume, $Y \sim 48 \pi T_{2}$.

We can therefore split $Y$ into its linear growth and absorb the rest into the tree level coupling. Putting everything together,

$$
\Delta_{a}=\left(\frac{\hat{\beta}_{a}}{3}-k_{a}\right) \pi T_{2}+\text { logarithmic. }
$$

The idea is now to use precisely the universal part in order to cancel the linear growth of the running part. In other words, we wish to impose conditions on the $\mathscr{N}=2$ subsector that survives in the $6 \mathrm{~d}$ limit of the theory, and whose KK and winding excitations from the $4 \mathrm{~d}$ perspective are the origin of the decompactification problem, such that it exactly cancels the growth of the universal part.

Indeed, if the $\mathscr{N}=2$ subsector of the theory is chosen such that $\hat{\beta}_{a}=3 k_{a}$ for all gauge group factors that we wish to retain at low energies, the corresponding couplings will exhibit at most a logarithmic dependence on the compactification volume. This works in terms of model building because the actual matter of the theory comes from $\mathscr{N}=1$ sectors, is moduli independent and controls the logarithmic running.

The conditions to be imposed at the $6 \mathrm{~d}$ limit of the theory can take a very simple form [30]. For instance, for an $\mathrm{SO}(2 n)$ gauge group factor at level one,

$$
N_{V}+2^{n-4} N_{S}=2 n+4,
$$

it is simply a relation between the number of massless states in the vectorial and spinorial representation of $\mathrm{SO}(2 n)$.

After cancellation of the liner volume term, the gauge couplings run logarithmically according to

$$
\frac{16 \pi^{2}}{g_{a}^{2}(\mu)}=k_{a} \frac{16 \pi^{2}}{g_{s}^{2}}+\beta_{a} \log \frac{M_{s}^{2}}{\mu^{2}}+\beta_{a}^{\prime} \log \left(\frac{2 e^{1-\gamma}}{3 \pi \sqrt{3}} \frac{M_{\mathrm{KK}}^{2}}{M_{s}^{2}}\right)+\ldots
$$

The beta function coefficient $\beta_{a}$ is determined in terms of the twisted $\mathscr{N}=1$ matter, while $\beta_{a}^{\prime}$ is due to a residual contribution of KK and winding towers of states from $\mathscr{N}=2$ exact, and the spontaneously broken $\mathscr{N}=2 \rightarrow 0, \mathscr{N}=4 \rightarrow 0, \mathscr{N}=4 \rightarrow 2$ sectors.

There have been several explicit heterotic constructions [30] of super no-scale models with chirality, which realise this scenario, in which the decompactification problem is absent as explained here. In a preliminary scan of $10^{8}$ models performed in [27], out of which $7 \times 10^{4}$ satisfy various modest conditions (such as chirality and the super no-scale property), it was found that some $40 \%$ of them do not suffer from the decompactification problem. It is therefore an interesting open model-building problem to construct theories with all desired properties, taking also into account the running of couplings to low energies. 
In closing, we would like to make a brief comment about quantum corrections to gravitational couplings in the presence of spontaneously broken supersymmetry. In particular, we wish to ask whether the Einstein-Hilbert term renormalises at 1-loop. In the case of unbroken supersymmetry, this question was answered by Kiritsis and Kounnas in the 90's in a beautiful work [50] that laid the ground for a systematic and powerful analysis of such cases. The result was that the $R$ term does not run nor does it receive any moduli dependent corrections at one loop.

This result was shown to hold also in the case of spontaneously broken supersymmetry [25]. For Heterotic theories the one loop correction vanishes identically, whereas type II theories have a constant 1-loop correction. This correction can be shown to be topological. In fact, it is related to the elliptic genus of the theory and can be shown to equal one fourth of the Euler characteristic of the compactification manifold, $\chi(X) / 4$.

Acknowledgements I would like to thank the Workshop organisers for their kind invitation, as well as C. Angelantonj, J. Rizos and M. Tsulaia for the very pleasurable collaborations that resulted in the main works summarised in this report.

\section{References}

[1] C. Kounnas and M. Porrati, "Spontaneous Supersymmetry Breaking in String Theory," Nucl. Phys. B 310 (1988) 355. doi:10.1016/0550-3213(88)90153-8

[2] S. Ferrara, C. Kounnas, M. Porrati and F. Zwirner, "Superstrings with Spontaneously Broken Supersymmetry and their Effective Theories," Nucl. Phys. B 318 (1989) 75. doi:10.1016/0550-3213(89)90048-5

[3] C. Kounnas and B. Rostand, "Coordinate Dependent Compactifications and Discrete Symmetries," Nucl. Phys. B 341 (1990) 641. doi:10.1016/0550-3213(90)90543-M

[4] P. H. Ginsparg and C. Vafa, "Toroidal Compactification of Nonsupersymmetric Heterotic Strings," Nucl. Phys. B 289 (1987) 414. doi:10.1016/0550-3213(87)90387-7

[5] H. Itoyama and T. R. Taylor, "Supersymmetry Restoration in the Compactified $\mathrm{O}(16) \times \mathrm{O}(16)$-prime Heterotic String Theory,” Phys. Lett. B 186 (1987) 129. doi:10.1016/0370-2693(87)90267-X

[6] J. J. Atick and E. Witten, "The Hagedorn Transition and the Number of Degrees of Freedom of String Theory,” Nucl. Phys. B 310 (1988) 291. doi:10.1016/0550-3213(88)90151-4

[7] D. Kutasov and N. Seiberg, "Number of degrees of freedom, density of states and tachyons in string theory and CFT," Nucl. Phys. B 358 (1991) 600. doi:10.1016/0550-3213(91)90426-X

[8] I. Antoniadis and C. Kounnas, "Superstring phase transition at high temperature," Phys. Lett. B 261 (1991) 369. doi:10.1016/0370-2693(91)90442-S

[9] I. Antoniadis, J. P. Derendinger and C. Kounnas, "Nonperturbative temperature instabilities in $\mathscr{N}=4$ strings," Nucl. Phys. B 551 (1999) 41 doi:10.1016/S0550-3213(99)00171-6 [hep-th/9902032].

[10] K. R. Dienes, "Modular invariance, finiteness, and misaligned supersymmetry: New constraints on the numbers of physical string states," Nucl. Phys. B 429 (1994) 533 doi:10.1016/0550-3213(94)90153-8 [hep-th/9402006].

[11] C. Angelantonj, C. Kounnas, H. Partouche and N. Toumbas, "Resolution of Hagedorn singularity in superstrings with gravito-magnetic fluxes," Nucl. Phys. B 809 (2009) 291 doi:10.1016/j.nuclphysb.2008.10.010 [arXiv:0808.1357 [hep-th]]. 
[12] C. Angelantonj, M. Cardella, S. Elitzur and E. Rabinovici, "Vacuum stability, string density of states and the Riemann zeta function,” JHEP 1102 (2011) 024 doi:10.1007/JHEP02(2011)024 [arXiv:1012.5091 [hep-th]].

[13] B. McClain and B. D. B. Roth, "Modular Invariance for Interacting Bosonic Strings at Finite Temperature," Commun. Math. Phys. 111 (1987) 539. doi:10.1007/BF01219073

[14] K. H. O’Brien and C. I. Tan, "Modular Invariance of Thermopartition Function and Global Phase Structure of Heterotic String,” Phys. Rev. D 36 (1987) 1184. doi:10.1103/PhysRevD.36.1184

[15] L. Alvarez-Gaume, P. H. Ginsparg, G. W. Moore and C. Vafa, “An O(16) x O(16) Heterotic String," Phys. Lett. B 171 (1986) 155. doi:10.1016/0370-2693(86)91524-8

[16] I. Florakis, C. Kounnas and N. Toumbas, "Marginal Deformations of Vacua with Massive boson-fermion Degeneracy Symmetry,” Nucl. Phys. B 834 (2010) 273 doi:10.1016/j.nuclphysb.2010.03.020 [arXiv:1002.2427 [hep-th]].

[17] C. Angelantonj, I. Florakis and M. Tsulaia, "Universality of Gauge Thresholds in Non-Supersymmetric Heterotic Vacua,” Phys. Lett. B 736 (2014) 365 doi:10.1016/j.physletb.2014.08.001 [arXiv:1407.8023 [hep-th]].

[18] A. E. Faraggi, C. Kounnas and H. Partouche, "Large volume susy breaking with a solution to the decompactification problem,” Nucl. Phys. B 899 (2015) 328 doi:10.1016/j.nuclphysb.2015.08.001 [arXiv:1410.6147 [hep-th]].

[19] I. Florakis, "Universality of radiative corrections to gauge couplings for strings with spontaneously broken supersymmetry," J. Phys. Conf. Ser. 631 (2015) no.1, 012079 doi:10.1088/1742-6596/631/1/012079 [arXiv:1502.07537 [hep-th]].

[20] C. Angelantonj, I. Florakis and M. Tsulaia, "Generalised universality of gauge thresholds in heterotic vacua with and without supersymmetry," Nucl. Phys. B 900 (2015) 170 doi:10.1016/j.nuclphysb.2015.09.007 [arXiv:1509.00027 [hep-th]].

[21] S. Abel, K. R. Dienes and E. Mavroudi, “Towards a nonsupersymmetric string phenomenology," Phys. Rev. D 91 (2015) no.12, 126014 doi:10.1103/PhysRevD.91.126014 [arXiv:1502.03087 [hep-th]].

[22] S. Abel, "A dynamical mechanism for large volumes with consistent couplings," JHEP 1611 (2016) 085 doi:10.1007/JHEP11(2016)085 [arXiv:1609.01311 [hep-th]].

[23] B. Aaronson, S. Abel and E. Mavroudi, "On interpolations from SUSY to non-SUSY strings and their properties," arXiv:1612.05742 [hep-th].

[24] C. Angelantonj, I. Florakis and M. Tsulaia, "Universality in radiative corrections for non-supersymmetric heterotic vacua,” PoS PLANCK 2015 (2016) 005.

[25] I. Florakis, "Gravitational Threshold Corrections in Non-Supersymmetric Heterotic Strings," Nucl. Phys. B 916 (2017) 484 doi:10.1016/j.nuclphysb.2017.01.016 [arXiv:1611.10323 [hep-th]].

[26] S. Abel and R. J. Stewart, "Exponential suppression of the cosmological constant in nonsupersymmetric string vacua at two loops and beyond," Phys. Rev. D 96 (2017) no.10, 106013 doi:10.1103/PhysRevD.96.106013 [arXiv:1701.06629 [hep-th]].

[27] I. Florakis and J. Rizos, "Chiral Heterotic Strings with Positive Cosmological Constant," Nucl. Phys. B 913 (2016) 495 doi:10.1016/j.nuclphysb.2016.09.018 [arXiv:1608.04582 [hep-th]].

[28] C. Kounnas and H. Partouche, "Super no-scale models in string theory," Nucl. Phys. B 913 (2016) 593 doi:10.1016/j.nuclphysb.2016.10.001 [arXiv:1607.01767 [hep-th]]. 
[29] C. Kounnas and H. Partouche, " $\mathscr{N}=2 \rightarrow 0$ super no-scale models and moduli quantum stability," Nucl. Phys. B 919 (2017) 41 doi:10.1016/j.nuclphysb.2017.03.011 [arXiv:1701.00545 [hep-th]].

[30] I. Florakis and J. Rizos, "A Solution to the Decompactification Problem in Chiral Heterotic Strings," Nucl. Phys. B 921 (2017) 1 doi:10.1016/j.nuclphysb.2017.05.002 [arXiv:1703.09272 [hep-th]].

[31] C. Angelantonj, I. Florakis and B. Pioline, "A new look at one-loop integrals in string theory," Commun. Num. Theor. Phys. 6 (2012) 159 doi:10.4310/CNTP.2012.v6.n1.a4 [arXiv:1110.5318 [hep-th]].

[32] C. Angelantonj, I. Florakis and B. Pioline, “One-Loop BPS amplitudes as BPS-state sums," JHEP 1206 (2012) 070 doi:10.1007/JHEP06(2012)070 [arXiv:1203.0566 [hep-th]].

[33] I. Florakis, “One-loop Amplitudes as BPS state sums,” PoS Corfu 2012 (2013) 101 [arXiv:1303.3788 [hep-th]].

[34] C. Angelantonj, I. Florakis and B. Pioline, "Rankin-Selberg methods for closed strings on orbifolds," JHEP 1307 (2013) 181 doi:10.1007/JHEP07(2013)181 [arXiv:1304.4271 [hep-th]].

[35] C. Angelantonj, I. Florakis and B. Pioline, “Threshold corrections, generalised prepotentials and Eichler integrals,” Nucl. Phys. B 897 (2015) 781 doi:10.1016/j.nuclphysb.2015.06.009 [arXiv:1502.00007 [hep-th]].

[36] I. Florakis and B. Pioline, “On the RankinĐSelberg method for higher genus string amplitudes," Commun. Num. Theor. Phys. 11 (2017) 337 doi:10.4310/CNTP.2017.v11.n2.a4 [arXiv:1602.00308 [hep-th]].

[37] W. Fischler and L. Susskind, “Dilaton Tadpoles, String Condensates and Scale Invariance,” Phys. Lett. B 171 (1986) 383. doi:10.1016/0370-2693(86)91425-5

[38] W. Fischler and L. Susskind, "Dilaton Tadpoles, String Condensates and Scale Invariance. 2.," Phys. Lett. B 173 (1986) 262. doi:10.1016/0370-2693(86)90514-9

[39] C. Condeescu, I. Florakis and D. Lust, "Asymmetric Orbifolds, Non-Geometric Fluxes and Non-Commutativity in Closed String Theory,” JHEP 1204, 121 (2012) doi:10.1007/JHEP04(2012)121 [arXiv:1202.6366 [hep-th]].

[40] C. Angelantonj, M. Cardella and N. Irges, "An Alternative for Moduli Stabilisation,” Phys. Lett. B 641 (2006) 474 doi:10.1016/j.physletb.2006.08.072 [hep-th/0608022].

[41] J. Scherk and J. H. Schwarz, "Spontaneous Breaking of Supersymmetry Through Dimensional Reduction,” Phys. Lett. B 82 (1979) 60. doi:10.1016/0370-2693(79)90425-8

[42] J. Scherk and J. H. Schwarz, "How to Get Masses from Extra Dimensions," Nucl. Phys. B 153 (1979) 61. doi:10.1016/0550-3213(79)90592-3

[43] E. Cremmer, S. Ferrara, C. Kounnas and D. V. Nanopoulos, "Naturally Vanishing Cosmological Constant in $\mathscr{N}=1$ Supergravity,” Phys. Lett. B 133 (1983) 61. doi:10.1016/0370-2693(83)90106-5

[44] R. Rohm, "Spontaneous Supersymmetry Breaking in Supersymmetric String Theories," Nucl. Phys. B 237 (1984) 553. doi:10.1016/0550-3213(84)90007-5

[45] I. Antoniadis, “A Possible new dimension at a few TeV,” Phys. Lett. B 246 (1990) 377. doi:10.1016/0370-2693(90)90617-F

[46] V. S. Kaplunovsky, “One Loop Threshold Effects in String Unification,” Nucl. Phys. B 307 (1988) 145 Erratum: [Nucl. Phys. B 382 (1992) 436] doi:10.1016/0550-3213(92)90193-F, 10.1016/0550-3213(88)90526-3 [hep-th/9205068]. 
[47] L. J. Dixon, V. Kaplunovsky and J. Louis, "Moduli dependence of string loop corrections to gauge coupling constants,” Nucl. Phys. B 355 (1991) 649. doi:10.1016/0550-3213(91)90490-O

[48] I. Antoniadis, E. Gava and K. S. Narain, "Moduli corrections to gravitational couplings from string loops,” Phys. Lett. B 283 (1992) 209 doi:10.1016/0370-2693(92)90009-S [hep-th/9203071].

[49] I. Antoniadis, E. Gava and K. S. Narain, "Moduli corrections to gauge and gravitational couplings in four-dimensional superstrings," Nucl. Phys. B 383 (1992) 93 doi:10.1016/0550-3213(92)90672-X [hep-th/9204030].

[50] E. Kiritsis and C. Kounnas, "Infrared regularization of superstring theory and the one loop calculation of coupling constants,” Nucl. Phys. B 442 (1995) 472 doi:10.1016/0550-3213(95)00156-M [hep-th/9501020].

[51] E. Kiritsis, C. Kounnas, P. M. Petropoulos and J. Rizos, "Solving the decompactification problem in string theory,” Phys. Lett. B 385 (1996) 87 doi:10.1016/0370-2693(96)00880-5 [hep-th/9606087].

[52] E. Kiritsis, C. Kounnas, P. M. Petropoulos and J. Rizos, "Universality properties of N=2 and N=1 heterotic threshold corrections,” Nucl. Phys. B 483 (1997) 141 doi:10.1016/S0550-3213(96)00550-0 [hep-th/9608034].

[53] P. M. Petropoulos and J. Rizos, "Universal moduli dependent string thresholds in Z(2) x Z(2) orbifolds,” Phys. Lett. B 374 (1996) 49 doi:10.1016/0370-2693(96)00230-4 [hep-th/9601037].

[54] M. Henningson and G. W. Moore, "Threshold corrections in K3 x T2 heterotic string compactifications,” Nucl. Phys. B 482 (1996) 187 doi:10.1016/S0550-3213(96)00549-4 [hep-th/9608145].

[55] E. Kiritsis and N. A. Obers, "Heterotic type I duality in D $<10$-dimensions, threshold corrections and D instantons,” JHEP 9710 (1997) 004 doi:10.1088/1126-6708/1997/10/004 [hep-th/9709058].

[56] H. P. Nilles and S. Stieberger, "String unification, universal one loop corrections and strongly coupled heterotic string theory,” Nucl. Phys. B 499, 3 (1997) doi:10.1016/S0550-3213(97)00315-5 [hep-th/9702110].

[57] P. Mayr and S. Stieberger, "Threshold corrections to gauge couplings in orbifold compactifications," Nucl. Phys. B 407 (1993) 725 doi:10.1016/0550-3213(93)90096-8 [hep-th/9303017].

[58] J. A. Harvey and G. W. Moore, “Algebras, BPS states, and strings,” Nucl. Phys. B 463 (1996) 315 doi:10.1016/0550-3213(95)00605-2 [hep-th/9510182].

[59] J. A. Harvey and G. W. Moore, “On the algebras of BPS states,” Commun. Math. Phys. 197 (1998) 489 doi:10.1007/s002200050461 [hep-th/9609017].

[60] C. P. Bachas, “Unification with low string scale,” JHEP 9811, 023 (1998) doi:10.1088/1126-6708/1998/11/023 [hep-ph/9807415].

[61] I. Antoniadis and C. Bachas, "Branes and the gauge hierarchy," Phys. Lett. B 450 (1999) 83 doi:10.1016/S0370-2693(99)00102-1 [hep-th/9812093]. 